\title{
Statistical Descriptors of Ocean Regimes From the Geometric Regularity of SST Observations
}

\author{
Silèye O. Ba ${ }^{a}$, Emmanuelle Autret ${ }^{\mathrm{b}}$, Bertrand Chapron $^{\mathrm{b}}$, and Ronan Fablet ${ }^{\mathrm{a}}$
}

\author{
a Lab-STICC, Université Européenne de Bretagne, Technopole Brest Iroise, Plouzané, France \\ ${ }^{\mathrm{b}}$ CERSAT, IFREMER, Technopole Brest Iroise, Plouzané, France
}

\begin{abstract}
:
In this paper we evaluate to which extent the activity of ocean fronts can be retrieved from the geometric regularity of ocean tracer observations. Applied to sea surface temperature (SST), we propose a method for the characterization of this geometric regularity from curvature-based statistics along temperature level- lines in front regions. To assess the effectiveness of the proposed descriptors, we used 6 years (from 2003 to 2008) of daily SST observations of the regions of Agulhas in the South of Africa, and of Malvinas off the southern Brazilian coast. These experiments stress the relevance of geometric regularity features of tracer observation at ocean surface to characterize seasonal variations in ocean regimes.
\end{abstract}

Keywords: Geosciences and remote sensing ; image analysis ; image classification, image processing ; ocean temperature ; oceans ; sea surface ; Barium ; radio frequency ; satellites

\section{Introduction}

Today, satellite passive imagery provides routine measurements of ocean color and sea surface temperature (SST) to offer global or local pictures of patterns at different scales, as small as $1 \mathrm{~km}$. These observations often reveal the richness, the complexity, as well as the high variability of the upper ocean biology and dynamics. Accordingly, the statistical properties of these scalar quantities, such as sea surface satellite-sensed concentration of phytoplankton and temperature, has long been thought to provide direct practical descriptions of the dynamical properties of the ocean circulation.

For common tools, such as spectral analysis, structure functions and probability density functions, the literature is immense. Since the first available satellite images, these techniques have been applied and used to retrieve the statistical characterization of the oceanic turbulence $[1,2]$. Using large swath satellite data, the observations can reveal a wide range of sizes and a variety of shapes, with a large occurrence of frontal systems. Consequently, this textural richness often leads to continuous spectral estimates that span a very wide range of scales. Variances of these different scales are further often found to follow constant power-law distributions. Yet, spectral forms may only be very weak constraints concerning the structure of the underlying flows, as coherent structures essentially sign in the phase information of the satellite snapshots [3, 4]. To gain more insights, the analysis of the geometry and complexity of delineated structures, for instance as derived from the 
analysis of level sets, can then be proposed to help the characterization and interpretation of the underlying flow properties. Indeed, line stretching and bending are generally driven by both local small scales and non local larger scales of the velocity fields [5]. Surface contour statistics can thus further help to better understand the internal organization of the flow, as earlier suggested by Lesieur and Sadourny in [6] and more recently rationalized by Lapeyre and Klein in [7] under the use of the surface quasi-geostrophy dynamics.

In this letter, we thus follow some recent attempts to characterize ocean turbulence based on numerical simulations (see [8]) to focus on the analysis of the regularity of satellite derived SST level-lines in highly dynamical ocean provinces, namely, the Brazil-Malvinas confluence zone and the Great Agulhas Current region. As implemented, we propose and test local curvature based descriptors to characterize SST regularity. Our objectives are then to experimentally assess the efficiency of these descriptors by measuring their ability to capture expected mesoscale seasonal variations. Accordingly, we thus further evaluate the relevance of the curvature statistics to perform a robust classification of ocean dynamical regimes.

The remainder of this paper is organized as follows. Section 2 presents the proposed SST image regularity descriptors. Experiments and results conducted to assess the effectiveness of the proposed descriptors are presented in Section 3. Final discussion and conclusion are given in Section 4 .

\section{$2 \quad$ SST Image Regularity extraction}

\section{$2.1 \quad$ Iso-temperature extraction}

As recently discussed in [8], it is not clear how solely spectral analysis can help in studying ocean surface turbulence, and more particularly help in characterizing the complexity of front strengths and alignments. An alternative effective means to better distinguish between the length and the scale of discontinuity near fronts, is to consider local curvature statistics along level-lines of the SST images. As understood, the characterization of the geometric regularity in front regions may involve relevant analysis both at local or nonlocal scales. Thus, for a scale based analysis, a pre-processing step involving an anisotropic diffusion with various scale parameters is applied to the SST images. We applied a diffusion by mean curvature motion (MCM) that guarantees the topology of the hierarchical image level set representation to be maintained [9]. The extraction of the level-lines of SST images is then based on the fast level-set transform proposed in [10]. The MCM smoothing of an image $u_{0}(x, y)$ is based on the resolution of the partial differential equation (PDE):

$$
\left\{\begin{array}{l}
u_{t}=|\nabla u| \operatorname{div}\left(\frac{\nabla u}{|\nabla u|}\right) \\
u(x, y, 0)=u_{0}(x, y)
\end{array}\right.
$$

where $u(x, y, t)$ is the image smoothed at the required scale $t, \nabla u$ is the image gradient. The gradient operator is defined as $\nabla u=\left(\begin{array}{l}u_{x} \\ u_{y}\end{array}\right)$ where $u_{x}$ is the partial derivative of the image with respect to the component $x$. |.| is the Euclidean norm, and $\operatorname{div}($.$) is the divergence operator. The scale parameter t$ gives the number of time the PDE in Eq.1 is applied to the original image.

\subsection{SST Image Regularity Descriptors}

For each SST image, we consider the main front which is the isoline supporting the highest gradient energy. Fig.1 gives an example of main front for the region of Agulhas. Descriptors of the geometric regularity of the front are then computed from the marginal distributions of the local curvatures along the curve. It might be stressed that as the considered descriptors are computed curvature/orientation statistics along SST level-lines, these descriptors do not depend on the strength of the gradient across the front. Therefore only information about the geometry of the front is captured. We followed three approaches extracting the local curvatures.

Local tangents derivatives: The curvature of the level lines can be computed from the variations of the 


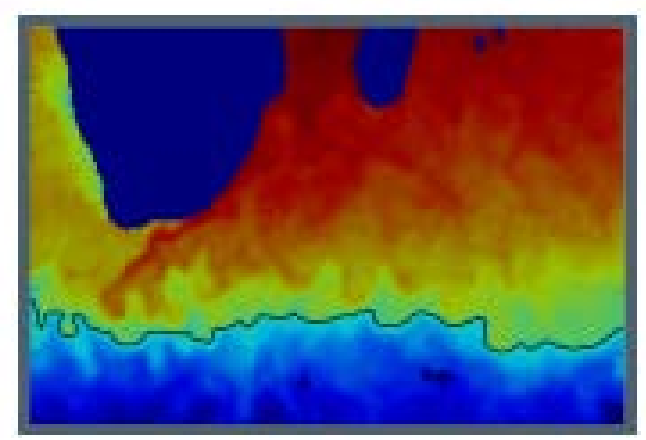

Figure 1: SST front sample: Agulhas main front defined as the isoline supporting the highest cumulated gradient magnitude.

directions of the level line local tangents. If we denote by $f(s)=(x(s), y(s))$ the level line parameterized by its curvilinear abscissa $s$, the orientation of the local tangent is given by $\theta(s)=\left|\arctan \left(\frac{\dot{y}(s)}{\dot{x}(s)}\right)\right|$ where $\dot{x}(s)$ is the derivative of $x$ with respect to $s$. An estimate of the local curvature can be obtained by the difference between the direction of the local tangents $\kappa(s)=|\theta(s)-\theta(s-1)|$.

Direct curvature computation: Rather than computing the derivatives of the local tangents orientation, the curvature of the level line $f(s)=(x(s), y(s))$ can be directly computed according to the formula $\kappa(s)=$ $\frac{\ddot{x}(s) \dot{y}(s)-\dot{x}(s) \ddot{y}(s)}{\left(\dot{x}^{2}(s)+\dot{y}^{2}(s)\right)^{\frac{3}{2}}}$ where $\ddot{x}(s)$ is the second derivative of $x(s)$ w.r.t. the curvilinear abscissa.

SST image divergence: The curvature of the level lines can also be derived as a partial differential operator applied to the SST image. This operator is given as the divergence of the normalized image gradient:

$$
\operatorname{curv}(\mathrm{u})=\operatorname{div}\left(\frac{\nabla u}{|\nabla u|}\right)=\frac{u_{x x} u_{y}^{2}-2 u_{x y} u_{x} u_{y}+u_{y y} u_{x}^{2}}{|\nabla u|^{3}}
$$

\section{$3 \quad$ Experiments}

In this section we describe the experiments we conducted to assess the relevance of the proposed descriptors for the statistical characterization of ocean regimes in highly dynamical ocean provinces, namely, the BrazilMalvinas confluence zone and the Great Agulhas Current region. We investigated to which extent the proposed descriptors can capture distinct seasonal variations.

\subsection{Evaluation Protocol}

A quantitative evaluation is carried out from the Remote Sensing System (REMSS) SST TMI-AMRE-OI database [11]. This database is publicly available at http://remss.com/sst/. These products consist in daily $25 \mathrm{~km}$ resolution global SST images recorded since the June 2002 to the present. The $25 \mathrm{~km}$ image resolution allows us to resolve only mesoscale phenomena. For our experiments we used data from 2003 to 2008 , with a 20 day buffer between seasons. For our two regions of interest the Agulhas region is localized in the South of Africa between $\left[-30^{\circ},-50^{\circ}\right]$ of latitudes and $\left[10^{\circ}, 70^{\circ}\right]$ of longitudes. The Malvinas area between $\left[-30^{\circ},-60^{\circ}\right]$ of latitudes and $\left[290^{\circ}, 330^{\circ}\right]$ of longitudes is off the Argentinian and Brazilian coasts. Let us point out that the seasons are denominated according to the South hemisphere terminology: September to October refers to Spring, December to March to Summer, March to June to Autumn and June to September to Winter.

With a view to assessing the relevance of the proposed descriptors for the characterization of ocean regimes in front regions through their efficiency to be seasonally discriminant, we tested for significant differences among seasons and evaluated season classification performances.

\subsection{Statistical variabilities of the descriptors}

The statistical significance of the proposed descriptors was evaluated using the following non parametric approach. The main motivation of these non-parametric tests is that they are valid even when the marginals of 


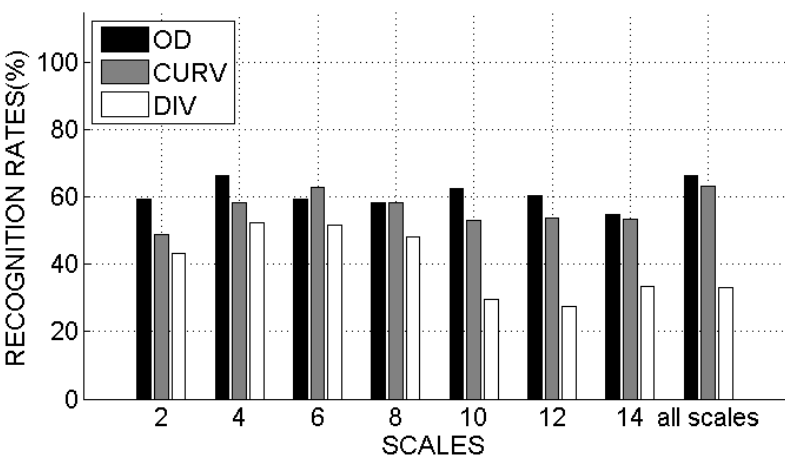

(a) Agulhas - NNR

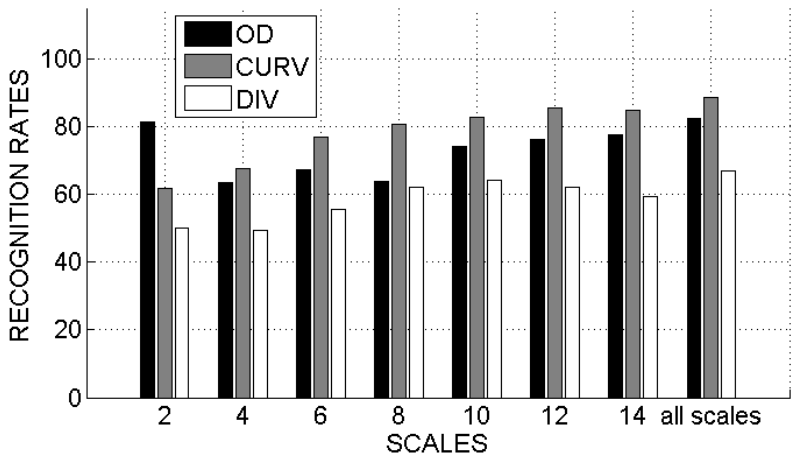

(c) Malvinas - NNR

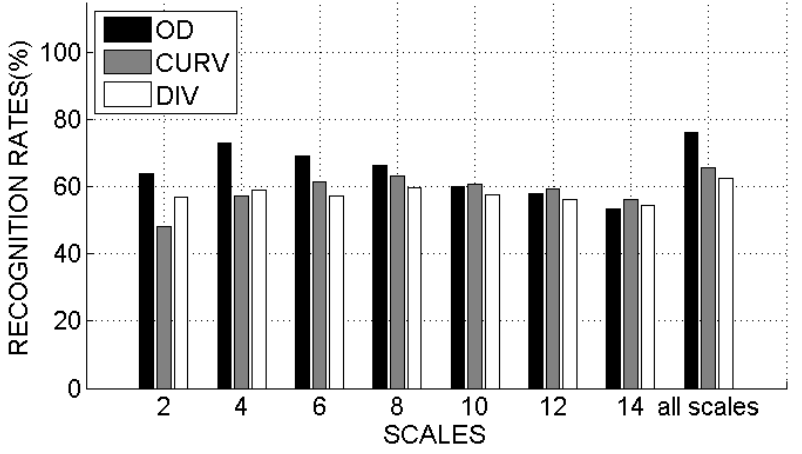

(b) Agulhas - RF

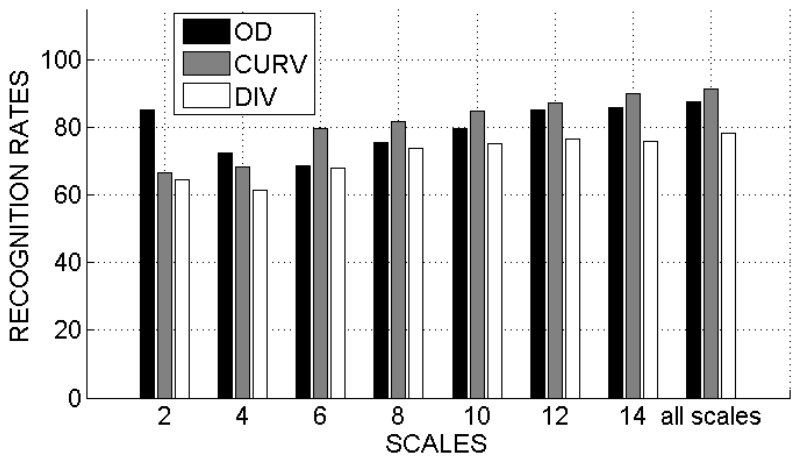

(d) Malvinas - RF

Figure 2: Average recognition rates in $\%$ using a nearest neighbors or a random forest classification method fed with the marginal of level line orientation difference (OD), the curvatures (CURV) and the image divergence (DIV). The item all scales corresponds to the situation where descriptors from all the image scales (2 to 14) are used as observation.

the considered descriptors do not conform to standard parametric distributions, typically Gaussian laws. Given two classes, $\mathcal{S}_{1}$ and $\mathcal{S}_{2}$, and associated SST samples characterized respectively by descriptors $p_{1, i}$ and $p_{2, j}$, we tested for the significance of the difference between the two classes according to the following criterion, inspired from the Fisher statistics,

$$
F=\frac{\rho\left(\mu_{1}, \mu_{2}\right)}{\sqrt{\frac{1}{\left|\mathcal{S}_{1}\right|} \sum_{i} \rho\left(p_{1, i}, \mu_{1}\right)+\frac{1}{\left|\mathcal{S}_{2}\right|} \sum_{j \in C_{2}} \rho\left(p_{2, j}, \mu_{2}\right)}}
$$

where $\mu_{1}$ and $\mu_{2}$ are the average descriptors within each class, and $\rho$, the Battacharya distance between two distributions $p=\left(p_{k}, k=1, . ., K\right)$ et $q=(q, k=1, . ., K)$, is defined as $\rho(p, q)=\sqrt{1-\sum_{k=1}^{K} \sqrt{p_{k}} \sqrt{q_{k}}}$. We used the Battacharya distance because it is well suited to compare histograms [12]. Note that similar performances were obtained when using the Kullback-Liebler divergence. The non-parametric test first evaluates the distribution of criterion (3) for the null hypothesis, i.e. the absence of meaningful differences, as follows. Given the set formed by the samples of the two classes denoted by $\mathcal{S}_{12}$, we sample Fisher statistics by generating two bootstrapped classes uniformly sampled from set $\mathcal{S}_{12}$. From these bootstrapped samples, we evaluate the partition function $P_{H_{0}}(F>\epsilon)$ of measure $F$ in Eq.3 under the null hypothesis. The two original classes are then regarded as significantly different if the likelihood that the associated discrimination measure $F_{1,2}$ has been generated from the null hypothesis is very low, i.e. if $p_{H_{0}}\left(F>F_{1,2}\right)<\epsilon$ were $\epsilon$ is typically set to 0.01 or 0.001. This statistical test can be seen as a Mantel test using the Fisher criterion [13].

Based on this non-parametric test, we showed that significant differences among seasons $\left(p\left(F>F_{1,2}\right)<\right.$ 0.001) were exhibited by all the proposed SST observations geometric regularity descriptors for the two selected regions. Thus, we can conclude that SST images regularity depicts significant seasonal differences in accordance 
with the seasonal front activities. This conclusion is in agreement with [14] which showed that the distribution of SST observations flow vorticities depict seasonal variations. It might be noted that using a parametric Student test led to the same conclusions.

\subsection{Categorization of ocean regimes from curvature statistics}

We further evaluated the discrimination power of the considered curvature statistics for the seasonal classification of ocean turbulence patterns. More precisely, given the dataset of SST images, each sample being assigned to a class, i.e. the season corresponding to the observation date, we apply supervised classification strategies from the proposed feature space. We used two classification methods: a generative method which makes easier the interpretation of the results, and a discriminative method known to depict greater classification performances.

The generative method proceeds as follows. For a given class, the geometric regularity of the class is modeled as the average feature, here marginals of curvature statistics, over the samples of the class. The classification of a new SST observation is achieved using a nearest neighbor criterion, according to the Battacharya distance. Note that similar performances were achieved for the Kullback-Liebler divergence. The nearest neighbor classification rule (NNR) assigns the class with the minimal distance between the curvature marginals of the classes and of the observation. We applied this generative classification strategy with a view to providing some insights on the relevance of the different types of descriptors. We also analyzed to which extent scaling effects were of interest. For the discriminative method, we considered random forests (RF) which are ensembles of classification trees [15]. Experiments not reported in this note also demonstrated that other discriminative classifiers such multi-layer perceptron (MLP) and a support vector machine (SVM) achieve similar performances [16]. The number of trees in the RF were estimated using cross-validation. A key advantage of learning scheme such as RF is that they directly allow to combine information (descriptors) at different scales to predict the seasonality. Labeled training samples are used to learn the classifier which, given the descriptors of an input test image predicts the corresponding season. Fig.2 reports, as a function of the processing scale, the classification performances achieved by the nearest neighbor and random forest classifiers with the descriptors given in Section 2.2. Curvature statistics issued from the local orientations of the SST level-lines clearly outperform those computed from the divergence operator. The lower recognition rate obtained with the latter might be explained by the numerical instability of the computation of this operator. These results (see Fig.2) advocate for a direct computation of the front local curvatures from the SST level lines. Besides, better classification performance are reported for the region of Malvinas compared to the region of Agulhas. The results in Fig.2 show that the characteristic scales are different for Agulhas and Malvinas. While for Agulhas the best performances are achieved for a scale of 6 , for Malvinas the best results are obtained for a scale of 14 . This might be explained by larger geometric structures in the region of Malvinas.

Fig.3 gives the performance of the nearest neighbor classifier and the random forest using the curvature descriptor. The results in this figure show that the RF achieves better performances than the nearest neighbor criterion. The improvements can be explained by intrinsic discriminative nature of RFs which are better suited to the discrimination task than the nearest neighbors criterion which was built without considering any discrimination criterion. When analyzing the results we can notice that for the two regions, performances are scale dependent, and there is no procedure to infer a priori the optimal scale value. This issue could be addressed by adopting an integrated multi-scale approach.

Fig,2, 3, and 4 also report the performances of a multi-scale strategy. These results show that, except for NNR classifier using the divergence features of the region of Agulhas, the multi-scale approach either performs similarly or outperforms the single scale approach. The better performances of the multi-scale approach can be explained by the intrinsically multi-scale nature of the underlying frontogenesis phenomenon associated with turbulent dynamics. Thus multi-scale information improves the characterization of the seasonal front activity. The performances of the nearest-neighbor classifier for the region of Agulhas where the single scale approach 


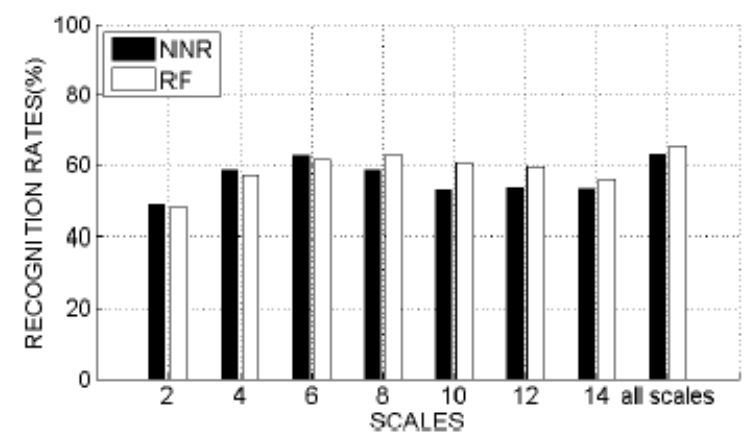

(a) Agulhas

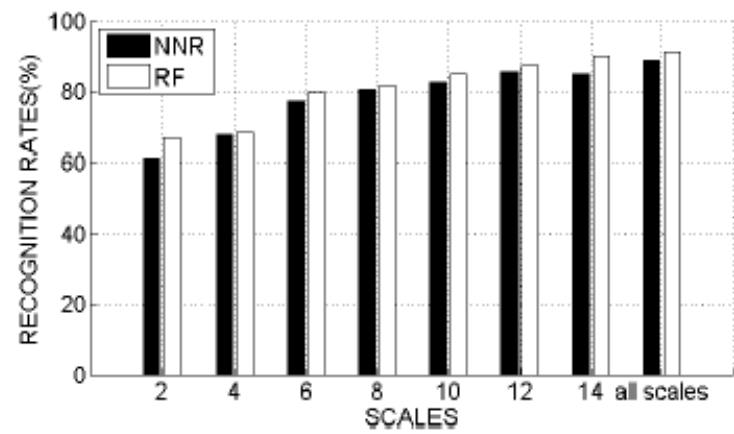

(b) Malvinas

Fig. 3. Average recognition rates in $\%$ for a $\mathrm{NNR}$ and an RF classification method using as observations| the marginal of the level line curvatures. 


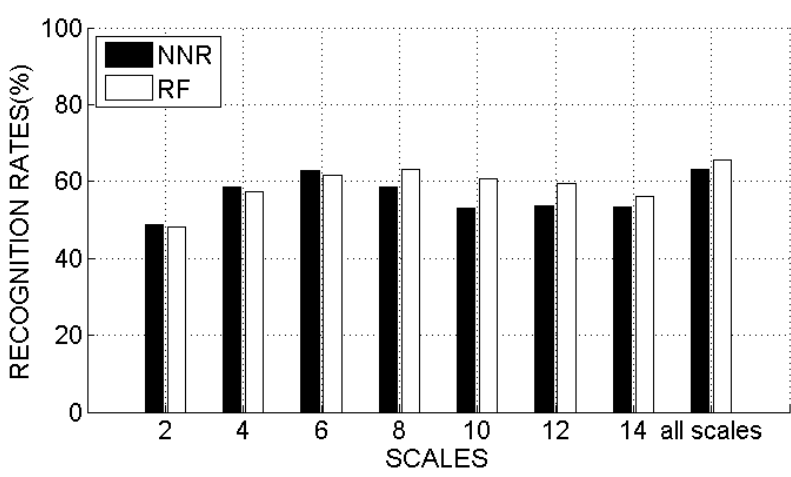

(a) Agulhas

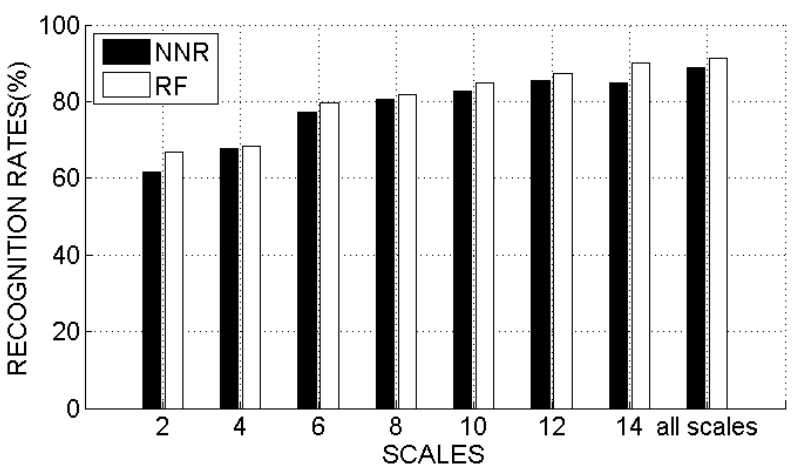

(b) Malvinas

Figure 3: Average recognition rates in $\%$ for a NNR and an RF classification method using as observations the marginal of the level line curvatures.

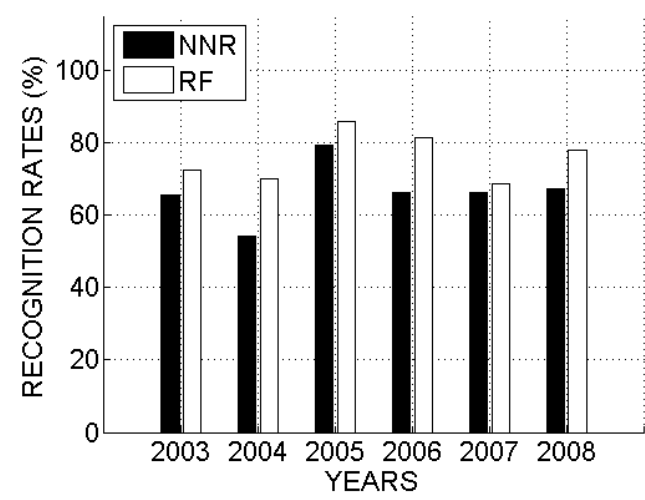

(a) Agulhas

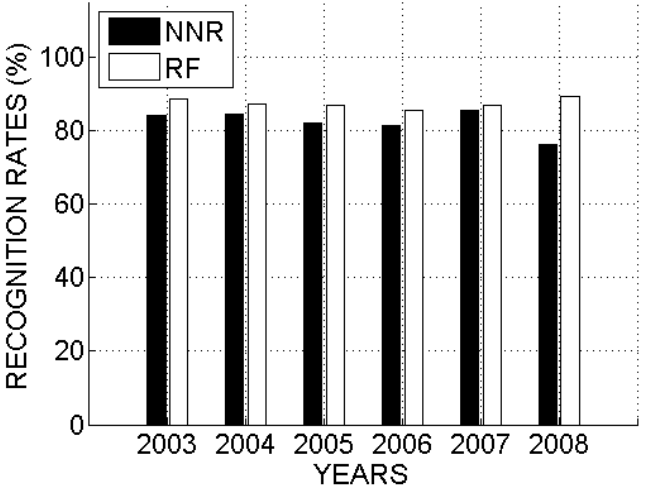

(b) Malvinas

Figure 4: Yearly multi-scale season classification rates in $\%$ for the NNR and the RF for regions of Agulhas and Malvinas. The RF systematically achieves better performance than the NNR method. However for a given method high yearly performance variations occur specially for the region of Agulhas.

outperformed the multi-scale approach may be explained by the multi-scale descriptor fusion procedure. Considering directly the average of the distance across scales may not be an optimal criterion, as the distance at one given scale may be dominant in the resulting average. The distance across scales could be defined in a better way by taking into account the final classification task using distance learning procedures as proposed in [17]. This would make the generative model more suited to classification task. However, the possibility to directly interpret the models, as shown in Fig.5, would be lost.

We also analyzed the performances of the multi-scale regularity-based methods at a yearly level and at a season level. Fig,4 reports the mean yearly classification performances from 2003 to 2006 . These results confirmed the better performance of the RF method over the NNR classifier. They also showed variability. Tab.1 and 2 give the average confusion tables for the region of Agulhas. These confusions tables show that Summer and Winter are better recognized (up to $85 \%$ for the RF method) and confusions mainly occur between neighboring seasons. The better performances obtained for Summer and Winter can be understood from an analysis of the cumulative distribution of the average marginal distribution for each season given in Fig.5. We can notice that Winter is the season for which the SST are the less regular because its marginal distribution exhibits the slowest decay. While Summer is the most regular season. These two seasons were, as expected, the two extremes situations in terms of geometric regularity resulting in greater discrimination performances. It might however be noted that despite the weakly apparent differences in the distribution of regularity features 


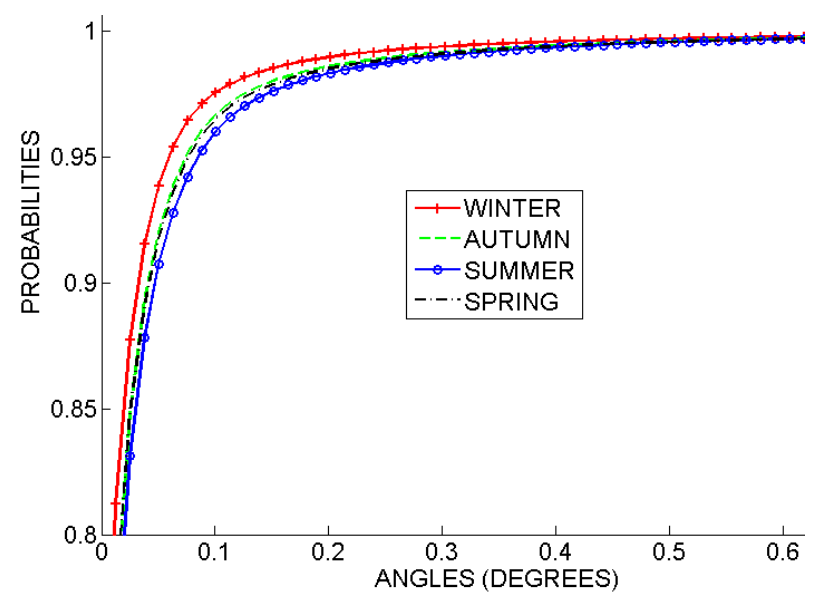

Figure 5: Local tangent orientation difference cumulative distributions.

\begin{tabular}{|l|c|c|c|c|}
\hline & Summer & Autumn & Winter & Spring \\
\hline Summer & 82.8 & 12.9 & 0 & 4.3 \\
\hline Autumn & 18.8 & 53.4 & 19.1 & 8.6 \\
\hline Winter & 0 & 2.6 & 78.5 & 18.9 \\
\hline Spring & 11.3 & 1.0 & 23.7 & 64.0 \\
\hline
\end{tabular}

Table 1: Average confusion in $\%$ for the multi-scale NNR for Agulhas. Better performances are achieved for summer and winter and confusion occurs mainly between neighboring seasons.

intermediate Spring and Autumn were also correctly discriminated with an average rate greater than $68 \%$ for RF method in Tab,2. These results indicate that Spring and Autumn also depict specific discriminative regularity features.

\section{Conclusions}

In this paper, we investigated the potential of satellite observations of ocean tracers to characterize ocean mesoscale turbulence regimes. Following numerical simulations of ocean turbulence [8], we evaluated descriptive statistics of the regularity of SST level-lines as descriptors of the underlying flow dynamics in active front regions. The reported experiments for 6 years of daily SST observations in two highly dynamical ocean provinces, namely, the Brazil-Malvinas confluence zone and the Great Agulhas Current region, clearly showed that, beyond spectral analysis, multiscale regularity statistics of SST level-lines relevantly captured the seasonal variabilities of frontogenesis activities.

These results advocate for further explorations of the potential of satellite observations of ocean surface, including high resolution SST observations, altimetry data and ocean colour, to refine and improve the proposed characterization of the mesoscale ocean turbulence regimes, and study its generalization to sub-mesoscale feature characterization. Also, as prospective future work, we will explore the use of such regularity-based statistical description as geometric priors in missing data interpolation of ocean observations, especially for the reconstruction of fine-scale textured structures which are poorly resolved by operational products $[18,19]$. 


\begin{tabular}{|l|c|c|c|c|}
\hline & Summer & Autumn & Winter & Spring \\
\hline Summer & 85.6 & 13.8 & 0 & 0.6 \\
\hline Autumn & 14.2 & 75.6 & 3.4 & 6.8 \\
\hline Winter & 0 & 4.8 & 84.3 & 10.9 \\
\hline Spring & 5.7 & 5.3 & 20.7 & 68.3 \\
\hline
\end{tabular}

Table 2: Average confusion in $\%$ for the multi-scale RF for Agulhas. Better performances are achieved for summer and winter and confusion occurs mainly between neighboring seasons.

\section{References}

[1] J. F. R. Gower, K. L. Denman, and R. J. Holyer, "Phytoplankton patchiness indicates the fluctuation spectrum of mesoscale oceanic structure," Nature, 1980.

[2] P. Y. Deschamps, R. Frouin, and L. Wald, "Satellite determinations of the mesoscale variability of the sea surface temperature," Physical Review Letter, pp. 864-870, 1981.

[3] L. Armi and P. Flament, "Cautionary remarks on the spectral interpretation of turbulent flows," Geophysical Research Letter, 1985.

[4] J. Isern-Fontanet, B. Chapron, G. Lapyere, and P. Klein, "Potential use of microwave sea surface temperatures for the estimation of ocean currents," Geophysical Research Letter, 2006.

[5] S. Girimaji and S. B. Pope, "Material-element deformation in isotropic turbulence," Journal of Fluid Mechanic, pp. 427-458, 1990.

[6] M. Lesieur and R. Sadourny, "Satellite-sensed turbulent ocean structure," Nature, 1981.

[7] G. Lapeyre and P. Klein, "Dynamics of the upper oceanic layers in terms of surface quasigeostrophy theory," Journal of Physical Oceanography, pp. 165-176, 2006.

[8] X. Capet, J. Isern-Fontanet, F. Colas, B. Chapron, and P. Klein, "Toward a practical characterization of submesoscale oceanic regimes," submitted to Journal of Physical Oceanography, 2011.

[9] L. Alvarez, F. Guichard, P.-L. Lions, and J.-M. Morel, "Axioms and fundamental equations of image processing," Archive for Rational Mechanics and Analysis, pp. 199-257, 1993.

[10] P. Monasse and F. Guichard, "Fast computation of a contrast-invariant image representation," IEEE Transactions on Image Processing, pp. 860-872, 2000.

[11] F. Wentz, P. Ashcroft, and C. Gentemann, "Post-launch calibration of the TMI microwave radiometer," IEEE Transactions on Geosciences and Remote Sensing, pp. 415-422, 2001.

[12] F. Lieseand and I. Vajda, "On divergences and informations in statistics and information theory," IEEE Transactions on Information Theory, pp. 4394-4412, 2006.

[13] N. Mantel and R. Valand, "A technique of nonparametric multivariate analysis," Biometrics, pp. 547-558, 1970.

[14] Q. Yang, B. Parvin, A. J. Mariano, E. H. Ryan, R. Evans, and B. O. Brown, "Seasonal and inter-annual studies of vortices in sea surface temperature data," International Journal of Remote Sensing, pp. 13711376, 2004.

[15] L. Breiman, "Random forests," Machine Learning, pp. 5-32, 2001. 
[16] R. Caruana and A. Niculescu-Mizil, "An empirical comparison of supervised learning algorithms using different performance metrics," International Conference on Machine Learning, 2006.

[17] A. Frome, Y. Singer, F. Sha, and J. Malik, "Learning globally consistent local distance functions for shape-based image retrieval and classification," International Conference On Computer Vision, 2007.

[18] R. Reynolds and T. M. Smith., "Improved global sea surface temperature analysis using optimum interpolation," Journal of Climate, 1994.

[19] J. L. Hoyer and J. Shea, "Optimal interpolation of sea surface temperature for the north sea and baltic sea," Journal of Marine Systems, pp. 176-189, 2007. 